TRANSACTIONS OF THE

AMERICAN MATHEMATICAL SOCIETY

Volume 361, Number 8, August 2009, Pages 4215-4232

S 0002-9947(09)04627-3

Article electronically published on March 4, 2009

\title{
GROWTH OF $L^{p}$ LEBESGUE CONSTANTS FOR CONVEX POLYHEDRA AND OTHER REGIONS
}

\author{
J. MARSHALL ASH AND LAURA DE CARLI
}

AbStract. For any convex polyhedron $W$ in $\mathbb{R}^{m}, p \in(1, \infty)$, and $N \geq 1$, there are constants $\gamma_{1}(W, p, m)$ and $\gamma_{2}(W, p, m)$ such that

$$
\gamma_{1} N^{m(p-1)} \leq \int_{\mathbb{T}^{m}}\left|\sum_{k \in N W} e(k \cdot x)\right|^{p} d x \leq \gamma_{2} N^{m(p-1)} .
$$

Similar results hold for more general regions. These results are various special cases of the inequalities

$$
\gamma_{1} N^{m(p-1)} \leq \int_{\mathbb{T}^{m}}\left|\sum_{k \in N B} e(k \cdot x)\right|^{p} d x \leq \gamma_{2} \phi(N),
$$

where $\phi(N)=N^{p(m-1) / 2}$ when $p \in\left(1, \frac{2 m}{m+1}\right), \phi(N)=N^{p(m-1) / 2} \log N$ when $p=\frac{2 m}{m+1}$, and $\phi(N)=N^{m(p-1)}$ when $p>\frac{2 m}{m+1}$, where $B$ is a bounded subset of $\mathbb{R}^{m}$ with non-empty interior.

\section{INTRODUCTION}

1.1. One dimension. In one dimension there is a very well known estimate

$$
\left\|\sum_{|k| \leq N} e(k x)\right\|_{1}=\int_{\mathbb{T}^{1}}\left|\sum_{|k| \leq N} e(k x)\right| d x=\int_{0}^{1}\left|\sum_{|k| \leq N} e(k x)\right| d x \simeq \frac{4}{\pi^{2}} \ln N,
$$

where $e(x)=e^{2 \pi i x}[\mathrm{Z}]$. This integral is called the Lebesgue constant. In higher dimensions there are as many Lebesgue constants as there are generalizations of an interval of integers, $\{-N,-N+1, \ldots, N\}$.

Fix $N$ and consider the operator $D$ defined by mapping a Lebesgue integrable function $f: \mathbb{T}^{1} \rightarrow \mathbb{C}$ to its $N$-th trigonometric partial sum $s_{N}(x)=$ $\sum_{|k| \leq N} \hat{f}(k) e(k x)$. This operator $D$ is realized by convolving $f$ with $\sum_{|k| \leq N} e(k x)$. For short,

$$
D: f \rightarrow s_{N}
$$

Received by the editors January 16, 2007 and, in revised form, August 3, 2007.

2000 Mathematics Subject Classification. Primary 42B15, 42A05; Secondary 42B08, 42A45.

Key words and phrases. Lebesgue constant, Dirichlet kernel, kernels for convex sets, kernels for polyhedra.

The first author's research was partially supported by a grant from the Faculty and Development Program of the College of Liberal Arts and Sciences, DePaul University.

(C)2009 American Mathematical Society Reverts to public domain 28 years from publication 
If we endow both the preimage and the image of $f$ with the $L^{1}\left(\mathbb{T}^{1}\right)$ norm, then the operator norm of $D,\|D\|_{L^{1} \rightarrow L^{1}}$, is defined to be $\sup _{\|f\|_{1}=1}\left\|s_{N}\right\|_{1}$. The HausdorffYoung inequality tells us that

$$
\left\|s_{N}\right\|_{1}=\left\|\sum_{|k| \leq N} e(k x) * f\right\|_{1} \leq\left\|\sum_{|k| \leq N} e(k x)\right\|_{1}\|f\|_{1} .
$$

Using $2 \nu \chi_{[-\nu, \nu]}$ in place of $f$ and letting $\nu \rightarrow \infty$ shows that $\left\|\sum_{|k| \leq N} e(k x)\right\|_{1}$ is the operator norm of $D$. Thus the operator norm is equal to the Lebesgue constant,

$$
\|D\|_{L^{1} \rightarrow L^{1}}=\left\|\sum_{|k| \leq N} e(k x)\right\|_{1} .
$$

This is the main reason for the importance of finding a good estimate for the Lebesgue constant. If we consider the same operation $D$, but consider $D$ as a mapping from $f \in L^{1}$ to $s_{N} \in L^{p}, p \geq 1$, then things proceed about the same as when $p$ was 1 . Young's inequality gives

$$
\left\|s_{N}\right\|_{p}=\left\|\sum_{|k| \leq N} e(k x) * f\right\|_{p} \leq\left\|\sum_{|k| \leq N} e(k x)\right\|_{p}\|f\|_{1},
$$

where $\|\cdot\|_{p}=\left(\int_{\mathbb{T}^{1}}|\cdot|^{p} d x\right)^{1 / p}$, and again using $2 \nu \chi_{[-\nu, \nu]}$ we arrive at a formula for the operator norm of $D$,

$$
\|D\|_{L^{1} \rightarrow L^{p}}=\left\|\sum_{|k| \leq N} e(k x)\right\|_{p}
$$

Because of this formula, the following lemma is also of interest.

\section{Lemma 1.}

$$
\int_{0}^{1}\left|\sum_{k=0}^{N-1} e(k x)\right|^{p} d u=\delta_{p} N^{p-1}+ \begin{cases}O_{p}\left(N^{p-3}\right) & \text { if } p>3 \\ O(\log N) & \text { if } p=3 \\ O_{p}(1) & \text { if } 1<p<3\end{cases}
$$

where the constant $\delta_{p}=\frac{2}{\pi} \int_{0}^{\infty}\left|\frac{\sin u}{u}\right|^{p} d u$. If $p=2 k$ is a positive even integer, then

$$
\delta_{2 k}=\frac{\left\langle\begin{array}{c}
2 k-1 \\
k
\end{array}\right)}{(2 k-1) !}
$$

where the central Eulerian number $\left\langle\begin{array}{c}2 k-1 \\ k\end{array}\right\rangle$ may be defined to be

$$
\sum_{\nu=0}^{k-1}(-1)^{\nu}\left(\begin{array}{c}
2 k \\
\nu
\end{array}\right)(k-\nu)^{2 k-1} \text {. }
$$


Most of this lemma appears elsewhere; one place is in the reference AAJRS. Only the formula (1.3) will be explained below.

1.2. Lebesgue constants for polyhedrons. If we let $W=[-1,1]$, then $W \subset$ $\mathbb{R}$, an interval, may be thought of as a one-dimensional convex polygon, and $\{-N,-N+1, \ldots, N\}$ may be thought of as $N W \cap \mathbb{Z}=\left\{y \in \mathbb{Z}: \frac{y}{N} \in W\right\}$, a dilate of $W$ by the scaling factor $N$. Let $f \in L^{1}\left(\mathbb{T}^{m}\right)$. An $m$-dimensional convex polyhedron $W$ gives rise to a sequence of partial sums $\left\{s_{N}\right\}$ according to the formula

$$
s_{N}(f)(x)=\sum_{k \in N W \cap \mathbb{Z}^{m}} \hat{f}(k) e(k \cdot x),
$$

where $k \in \mathbb{Z}^{m}, x \in \mathbb{T}^{m}$, and $\hat{f}(k)=\int_{\mathbb{T} m} f(x) e(-k \cdot x) d x$. Just as in the onedimensional situation, we again find that for $N$ fixed and $D$ defined by

$$
D: f \rightarrow s_{N}
$$

we have for $p \geq 1$,

$$
\|D\|_{L^{1} \rightarrow L^{p}}=\left\|\sum_{k \in N W \cap \mathbb{Z}^{m}} e(k x)\right\|_{p}
$$

Let

$$
D_{N W}(x)=\sum_{k \in N W \cap \mathbb{Z}^{m}} e(k x) .
$$

There naturally occur two problems: good estimates for $\int_{\mathbb{T}^{m}}\left|D_{N W}(x)\right| d x$ and good estimates for $\int_{\mathbb{T}^{m}}\left|D_{N W}(x)\right|^{p} d x$ for $p>1$. The first problem has been very nicely solved by Belinsky.

Theorem 1. For any convex $m$-dimensional polyhedron $W$ in $\mathbb{R}^{m}$ and $N \geq 1$, there are constants $\gamma_{1}$ and $\gamma_{2}$ such that

$$
\gamma_{1}(W) \log ^{m}(1+N) \leq\left\|D_{N W}\right\|_{1}=\int_{\mathbb{T}^{m}}\left|D_{N W}(x)\right| d x \leq \gamma_{2}(W) \log ^{m}(1+N) .
$$

From now on we assume that $p>1$ and focus on the question of getting good estimates for

$$
\int_{\mathbb{T}^{m}}\left|D_{N W}(x)\right|^{p} d x .
$$

First note that if $W=[-1,1]^{m}$, then

$$
D_{N W}(x)=\sum_{k \in N W \cap \mathbb{Z}^{m}} e\left(k_{1} x_{1}\right) e\left(k_{2} x_{2}\right) \cdots e\left(k_{m} x_{m}\right)=\prod_{j=1}^{m} \sum_{k_{j}=-N}^{N} e\left(k_{j} x_{j}\right),
$$

So

$$
\begin{aligned}
\int_{\mathbb{T}^{m}}\left|D_{N W}(x)\right|^{p} d x & =\prod_{j=1}^{m} \int_{0}^{1}\left|\sum_{k_{j}=-N}^{N} e\left(k_{j} x_{j}\right)\right|^{p} d x_{j} \\
& =\left(\int_{0}^{1}\left|\sum_{k=-N}^{N} e(k x)\right|^{p} d x\right)^{m} \\
& =\delta_{p}^{m} 2^{m(p-1)} N^{m(p-1)}+o\left(N^{m(p-1)}\right) .
\end{aligned}
$$

This motivated the following theorem. 
Theorem 2. For any $p>1$ and convex $m$-dimensional polyhedron $W$ in $\mathbb{R}^{m}$ and $N \geq 1$, there are constants $\gamma_{1}$ and $\gamma_{2}$ such that

$$
\left\|D_{N W}\right\|_{p}^{p}=\int_{\mathbb{T}^{m}}\left|D_{N W}(x)\right|^{p} d x \leq \gamma_{2}^{p}(W, m, p) N^{m(p-1)}
$$

and

$$
\gamma_{1}^{p}(W, m, p) N^{m(p-1)} \leq\left\|D_{N W}\right\|_{p}^{p} .
$$

For short,

$$
\gamma_{1} N^{m\left(1-\frac{1}{p}\right)} \leq\left\|D_{N W}\right\|_{p} \leq \gamma_{2} N^{m\left(1-\frac{1}{p}\right)} .
$$

If the dimension is $m=2$, then inequality (1.7) has already been established, first for $p=1[\mathrm{YY}]$ and then for all $p>1[\mathrm{~A}$. The constants were more precise and the proofs were much more direct, but the proofs were number theoretic and difficult, and we do not know how to extend them to higher dimensions. Part of the argument given here will show that the result of $[\mathrm{A}$ easily leads to the reverse inequality (1.8) also when $m=2$. Another interesting proof for $m=2$ and $p=1$ is in $\mathrm{NP}$.

1.3. Lebesgue constants for more general sets. We will also consider the more general case when $W$ is allowed to have a more general shape; for example, when $W$ is replaced by a set $B \subset \mathbb{R}^{m}$ that is bounded and has non-empty interior. When $p=1$, the expected lower bound estimate of $\gamma \log ^{m}(1+N)$ is valid, but the corresponding upper value is larger. For example, when $B$ is an $m$-dimensional ball of radius $N$, then it turns out that

$$
\int_{T^{m}}\left|\sum_{|k| \leq N} e(k \cdot x)\right| d x \asymp N^{\frac{m}{2}-\frac{1}{2}},
$$

where $A \asymp N^{\alpha}$ means that $A / N^{\alpha}$ is bounded above and below.

Concerning the upper bounds in this generality, we have two results:

Theorem 3. For each $m \geq 2, p \geq 2$, and bounded set $B \subset \mathbb{R}^{m}$ with non-empty interior, there is a constant $\gamma_{2}$ such that the upper bound estimate

$$
\int_{T^{m}}\left|D_{N B}(x)\right|^{p} d x \leq \gamma_{2}^{p} N^{m(p-1)}
$$

holds,

and

Theorem 4. For each $m \geq 2, p \in(1,2]$, and bounded set $B \subset \mathbb{R}^{m}$ with non-empty interior, there is a constant $\gamma_{1}$ such that the lower bound estimate

$$
\int_{T^{m}}\left|D_{N B}(x)\right|^{p} d x \geq \gamma_{1}^{p} N^{m(p-1)}
$$

holds.

There remain two cases for general sets that we have not yet resolved. 
(1) The question of an upper estimate for

$$
\left\|D_{N B}\right\|_{p}=\left(\int_{\mathbb{T}^{m}}\left|\sum_{k \in N B \cap \mathbb{Z}^{m}} e(k \cdot x)\right|^{p} d x\right)^{1 / p}
$$

when $p \in(1,2)$ and

(2) the question of a lower estimate for the same integral when $p>2$.

We can only provide partial results in these two cases. Looking at the calculation (1.6) above, in both cases we would like to get an estimate of the form $c N^{m(1-1 / p)}$. In case (1), this is not true. We calculate below that if $B$ is the $m$-dimensional unit ball so that $N B=\left\{x \in \mathbb{R}^{m}:|x| \leq N\right\}$, then for $p \in\left(1, \frac{2 m}{m+1}\right)$ and this choice of $B$, we get the larger sharp lower estimate of $\gamma_{1} N^{\frac{m-1}{2}}=\gamma_{1} N^{m(1-1 / p)+\delta}$, where $\delta=m\left(\frac{1}{p}-\frac{m+1}{2 m}\right)$ is positive since $1 / p \in\left(\frac{m+1}{2 m}, 1\right)$. Interestingly, the exponent of $N$ is constant as $p$ varies from 1 to $\frac{2 m}{m+1}$.

\section{Theorem 5.}

$$
\left\|D_{N\{|x| \leq 1\}}\right\|_{p} \geq\left\{\begin{array}{cc}
\gamma_{1} N^{\frac{m-1}{2}}=\gamma_{1} N^{m(1-1 / p)+\delta} & \text { for } 1 \leq p<\frac{2 m}{m+1}, \\
\gamma_{1} N^{m(1-1 / p)} \ln ^{1 / p} N & \text { for } p=\frac{2 m}{m+1}, \\
\gamma_{1} N^{m(1-1 / p)} & \text { for } \frac{2 m}{m+1}<p \leq 2,
\end{array}\right.
$$

where $\delta=m\left(\frac{1}{p}-\frac{m+1}{2 m}\right), \gamma_{1}=\gamma_{1}(m, p)$.

To what extent this new candidate for an upper bound is actually extremal for all bounded sets with non-empty interior remains an open question.

In case (2), we get the hoped-for sharp (since $B$ may be the cuboid $[-1,1]^{m}$; see (1.6) above) best lower estimate of $c N^{m(1-1 / p)}$, but only by additionally assuming a small amount of regularity for the boundary of $B$. For this, we get inspiration from the paper of Liflyand $\left[\mathrm{L}\right.$. (See also $\left[\mathbf{I},\left[\underline{S h}\right.\right.$, and $[\mathrm{Y}]$.) Let $A_{N W}$ be the union of all cubes of side 1 whose centers are at the points of $N W \cap \mathbf{Z}^{m}$. We say that $W$ satisfies the $M_{N^{-}}$condition (the $M$ is for Minkowski) if

$$
\lim _{N \rightarrow \infty} \frac{\left|A_{N W} \triangle N W\right|}{N^{m}}=0,
$$

where the symmetric difference operator is defined by $A \triangle B=(A \backslash B) \cup(B \backslash A)$. In other words, the measure of $A_{N W} \triangle N W$ is $o\left(N^{m}\right)$. If $W$ is bounded with non-empty interior, then there are on the order of $N^{m}$ points in $N W$, and so we can then express that $M_{N}$-condition by saying that the measure of $A_{N W} \triangle N W$ must be "little oh" version of the number of lattice points in $N W$.

Theorem 6. If the bounded set $B \subset \mathbb{R}^{m}$ has non-empty interior and if $B$ satisfies the $M_{N}$-condition, then for $p \geq 2$ there holds the lower bound estimate

$$
\gamma_{1} N^{m(1-1 / p)} \leq\left\|D_{N B}\right\|_{p}
$$

for some constant $\gamma_{1}(B, m, p)$.

Another open question is whether the boundary condition (1.10) is necessary. The most optimistic set of conjectures might be that for bounded $B$ with non-empty interiors, $N^{m(1-1 / p)}$ is the size of the lower estimate for all $p, 1<p<\infty$, and also the size of the upper estimate for $p \in\left(\frac{2 m}{m+1}, \infty\right)$, while $N^{m(1-1 / p)+m\left(\frac{1}{p}-\frac{m+1}{2 m}\right)}=$ 
$N^{\frac{m-1}{2}}$ is the size of the upper estimate when $p \in\left(1, \frac{2 m}{m+1}\right)$ and $N^{\frac{m-1}{2}} \ln ^{\frac{m+1}{2 m}} N$ is the size of the upper estimate when $p=\frac{2 m}{m+1}$. In other words, the sphere is extremal for $p \in\left(1, \frac{2 m}{m+1}\right]$ and the cuboid is extremal for $p>\frac{2 m}{m+1}$.

Our intuition for this conjecture is that spheres and rectangles are extremal with respect to curvature, the former being everywhere curved and the latter nowhere curved. So it is at least plausible that for each value of $p$ one or the other should provide the largest or smallest Lebesgue constant growth rate.

In the $L^{1}$ case, results for all convex sets in all dimensions are already known. See chapter 6 of $[\mathrm{L}$ for this.

A small result of fairly great generality that does not seem to be well known is the M. Riesz Projection Theorem for a general half space. This theorem is the obvious generalization of the very well known fact that the Riesz transform of an $L^{p}\left(\mathbb{T}^{m}\right)$ function is also in $L^{p}$.

Theorem 7. Fix $\alpha \in \mathbb{R}^{m}$ and $b \in \mathbb{R}$. The associated hyperplane

$$
L=\left\{x \in \mathbb{R}^{m}: x \cdot \alpha=d\right\}
$$

divides $\mathbb{Z}^{m}$ into two halfspaces,

$$
H^{+}=\left\{k \in \mathbb{Z}^{m}: k \cdot \alpha \geq d\right\} \text { and } H^{-}=\left\{k \in \mathbb{Z}^{m}: k \cdot \alpha<d\right\} .
$$

Then if $f \in L^{p}\left(T^{m}\right), p>1$, we have

$$
\left\|\sum_{k \in H^{+}} \hat{f}(k) e(k \cdot x)\right\|_{p} \leq \frac{p^{2}}{p-1}\|f\|_{p} \text { and }\left\|\sum_{k \in H^{-}} \hat{f}(k) e(k \cdot x)\right\|_{p} \leq \frac{p^{2}}{p-1}\|f\|_{p} .
$$

One might think that replacing $\left\{k: k_{1} \geq 0\right\}$ by $\{k: \alpha \cdot k \geq d\}$ would lead to a serious number-theoretic problem. Fortunately, it does not. See the proof of Theorem 7 below.

We will prove all results but one in the next section. The last section will consist of the proof of Theorem 5 .

In several of the proofs we implicitly assume that the origin is interior to the polyhedron $W$ or the bounded set $B$. The general case may be reduced to the case where the origin is interior to the polyhedron or bounded set by making an initial translation. Such a translation may introduce an error term of lower order, but will not affect the final result. We will omit the details.

\section{Most of THE PRoOfs}

We begin with a discussion of Lemma 1. As we already mentioned, everything except the evaluation of $\delta_{2 k}$ appears in AAJRS. To evaluate $\delta_{2 k}$, let $N(x)$ be the characteristic function of $[0,1)$ and $N_{2 k}=N_{1} * N_{1} * \cdots * N_{1}$ be the $2 k$-fold convolution of $N_{1}$. The function $N_{m}(x)$ is called the $m$-th order cardinal $B$-spline. Then $\widehat{N_{2 k}}(x)=\left(\widehat{N_{1}}(x)\right)^{2 k}$, so setting $x=k$ in the double inversion relation $N_{2 k}(x)=\left(\widehat{N_{2 k}}(\omega)\right)^{\vee}(x)$ shows that

$$
N_{2 k}(k)=\frac{1}{\pi} \int_{-\infty}^{\infty}\left(\frac{\sin x}{x}\right)^{2 k} d x=\frac{2}{\pi} \int_{0}^{\infty}\left|\frac{\sin x}{x}\right|^{2 k} d x
$$


whereas on page 191 of [C] a direct computation shows $N_{2 k}(k)$ to be equal to the sum (1.4) divided by $(2 k-1)$ !. Finally, the identification of the sum (1.4) as the central Eulerian number appears in GKP.

Remark 1. The left hand side of relation (1.2) can also be evaluated exactly by expanding and using orthogonality when $p=2 k$, and this leads to the polynomial relation

$$
\sum_{\nu=0}^{(N-1) k} a_{N k}(\nu)^{2}=\frac{\left\langle\begin{array}{c}
2 k-1 \\
k
\end{array}\right)}{(2 k-1) !} N^{2 k-1}+O\left(N^{2 k-3}\right)
$$

where the $a_{N k}(\nu)$ are defined by

$$
\left(1+x+x^{2}+\cdots+x^{N-1}\right)^{k}=\sum_{\nu=0}^{(N-1) k} a_{N k}(\nu) x^{\nu} .
$$

This is immediate from the above result and the calculation

$$
\begin{aligned}
\left(\sum_{\nu=0}^{N-1} e(\nu x)\right)^{k}\left(\sum_{\nu=0}^{N-1} e(-\nu x)\right)^{k} & =\sum_{\nu=0}^{(N-1) k} a_{N k}(\nu) e(\nu x) \sum_{\nu=0}^{(N-1) k} a_{N k}(\nu) e(-\nu x) \\
& =\sum_{\nu=0}^{(N-1) k} a_{N k}(\nu)^{2}+\sum_{j \neq 0} C_{j} e(j x) .
\end{aligned}
$$

Actually it appears that $\int_{0}^{1}\left|\sum_{\nu=0}^{N-1} e(\nu x)\right|^{2 k} d x$ is in general an odd polynomial in $N$ of degree $2 k-1$. Relations (1.2) and (1.3) provide a formula for the leading coefficient of this polynomial. It might be interesting to find formulas for the other coefficients.

We turn our attention to establishing the upper bound inequality (1.7). If $W$ is a convex $m$-dimensional polyhedron in $\mathbb{R}^{m}$ and $N \geq 1$, then $W$ is a bounded set with a non-empty interior. A bounded subset $B \subset \mathbb{R}^{m}$ with non-empty interior has two geometric properties. Namely, there are constants $d(B)$ and $D(B)$ so that

$$
d N^{m} \leq\left|N B \cap \mathbb{Z}^{m}\right|
$$

and

$$
N B \subset D[-N, N]^{m}=[-D N, D N]^{m}
$$

We will use three lemmas, all of which are essentially from the proof of Theorem 1 on pages 404 and 405 of [TB].

For $x \in \mathbb{T}^{m}$ and $f \in L\left(\mathbb{T}^{m}\right)$, we define the $m$-dimensional de la Vallée-Poussin mean to be

$$
\tau_{2 N}(f)=\sum_{S \in P(\mathcal{M})} \sum_{\substack{N<\left|k_{j}\right| \leq 2 N, j \in S \\\left|k_{j}\right| \leq N, j \in \mathcal{M} \backslash S}}\left(\prod_{j \in S} \frac{2 N+1-\left|k_{j}\right|}{N-1}\right) \hat{f}(k) e(k \cdot x),
$$

where $P(\mathcal{M})$ is the power set of $\mathcal{M}=\{1,2, \ldots, m\}$. The spectrum of a trigonometric polynomial $\sum c_{k} e(k \cdot x)$ is the set of $k \in \mathbb{Z}^{m}$ for which $c_{k} \neq 0$. 
Lemma 2. Let $\tau_{2 N}(f)$ be the $m$-dimensional de la Vallée-Poussin mean of $f \in$ $L\left(T^{m}\right)$ and let $W$ satisfy conditions (2.1) and (2.2). Then

(1) The cardinality of the spectrum of $\tau_{2 D N}(f)$ is $O\left(N^{m}\right)$,

$$
s_{N W}(f)=s_{N W}\left(\tau_{2 D N}(f)\right),
$$

and

(3) for all $p \geq 1$,

$$
\left\|\tau_{2 D N}(f)\right\|_{p} \leq 3^{m}\|f\|_{p} .
$$

Proof. To make sense of $\tau_{2 D N}, D$ must be an integer, so if necessary simply replace $D$ by $\lceil D\rceil$. Then (1) is immediate from the definition of $\tau_{2 D N}$. For (2), note from the definition of $\tau$ that when all $\left|k_{j}\right| \leq D N, S=\varnothing$ and the multiplier of $\hat{f}(k) e(k \cdot x)$ is 1. We now establish (3). For $f \in L(\mathbb{T})$, the de la Vallée-Poussin mean $\tau_{2 n}(f)$ is defined to be $\sum_{|k| \leq n} \hat{f}(k) e(k x)+\sum_{n<|k| \leq 2 n}\left(\frac{2 n+1-|k|}{n+1}\right) \hat{f}(k) e(k x)$. From this we see that

$$
\tau_{2 n}(f)=\frac{2 n+1}{n+1} \sigma_{2 n}(f)-\frac{n}{n+1} \sigma_{n}(f),
$$

where the Cesàro mean $\sigma_{n}(f)$ corresponds to convolution with a positive kernel $k_{n}$. For $p \geq 1,\left\|\sigma_{n}(f)\right\|_{p} \leq\left\|k_{n}\right\|_{1}\|f\|_{p}=\left(\int_{0}^{1} k_{n}\right)\|f\|_{p}=\|f\|_{p}$. From this and (2.6) it follows that

$$
\left\|\tau_{2 n}(f)\right\|_{p} \leq 3\|f\|_{p} .
$$

Define $\tau_{2 D N}^{j}(f), f \in L\left(\mathbb{T}^{m}\right)$, to be the de la Vallée-Poussin mean of $f$ thought of as a function of $x_{j}$ with the other $m-1$ coordinates of $x$ fixed. We find that $\tau_{2 D N}(f)=$ $\tau_{2 D N}^{m}\left(\tau_{2 D N}^{m-1}\left(\ldots\left(\tau_{2 D N}^{1}(f)\right) \ldots\right)\right)$. This and inequality (2.7) lead to (3).

Lemma 3. Let $p \in(0,2]$ and let $T(x), x \in \mathbb{T}^{m}$, be a trigonometric polynomial with spectrum of cardinality $n$. Then for $q>p$,

$$
\|T\|_{q} \leq \gamma(m, p, q) n^{\frac{1}{p}-\frac{1}{q}}\|T\|_{p} .
$$

This is proved on page 131 of $\mathrm{TB}$. We refer to it as the change of norm lemma. It is a sort of reverse Hölder's inequality since $\|T\|_{p} \leq\|T\|_{q}$ is almost immediate from Hölder's inequality.

Lemma 4. Let $K$ be an arbitrary polyhedron in $\mathbb{R}^{m}$. Then there is a constant $\gamma$ depending only on $K$, such that for all $n \in N$ and for all $p \in(1, \infty)$ there holds

$$
\left\|s_{N K}(f)\right\|_{p} \leq \gamma\left(1+\frac{4 p^{2}}{p-1}\right)^{m}\|f\|_{p} .
$$

This is part of Corollary 2.4.5 on page 56 of [TB]. This is proved carefully, but we would like to fill in a detail at one point. The first step of the proof is to show that if $f \in L^{p}\left(\mathbb{T}^{m}\right)$ and if $H$ is a half space of $\mathbb{Z}^{m}$ having a hyperplane through the origin as its boundary $\left(H=\left\{k \in \mathbb{Z}^{m}: k \cdot \alpha \geq 0\right\}\right.$ for some $\left.\alpha \in \mathbb{R}^{m}\right)$ or as the boundary of its complement $\left(H=\left\{k \in \mathbb{Z}^{m}: k \cdot \alpha<0\right\}\right.$ for some $\left.\alpha \in \mathbb{R}^{m}\right)$, then

$$
\left\|\sum_{k \in H} f(k) e(k \cdot x)\right\|_{p} \leq \frac{p^{2}}{p-1}\|f\|_{p} .
$$


This is Theorem 2.4.3, the M. Riesz Theorem on Projections, on page 54 of [TB]. The second step is to assert that the same result holds if the bounding hyperplane does not pass through the origin. The third step is to decompose a general polyhedron into convex polyhedra of $m+1$ sides. The last step is to intersect half spaces in a clever way in order to get the result for a convex polyhedron of $m+1$ sides. Steps 1, 3, and, 4 are carried out carefully on pages $54-57$ of [TB]. We will now do step 2 by proving Theorem 7

Proof. We are given $f \in L^{p}\left(T^{m}\right), p>1$, fixed $\alpha \in \mathbb{R}^{m}$, and fixed $b \in \mathbb{R}$. We must show

$$
\left\|\sum_{k \in H^{+}} \hat{f}(k) e(k \cdot x)\right\|_{p} \leq \frac{p^{2}}{p-1}\|f\|_{p} \text { and }\left\|\sum_{k \in H^{-}} \hat{f}(k) e(k \cdot x)\right\|_{p} \leq \frac{p^{2}}{p-1}\|f\|_{p},
$$

where the hyperplane $L=\left\{x \in \mathbb{R}^{m}: x \cdot \alpha=d\right\}$ divides $\mathbb{Z}^{m}$ into the two halfspaces, $H^{+}=\left\{k \in \mathbb{Z}^{m}: k \cdot \alpha \geq d\right\}$ and $H^{-}=\left\{k \in \mathbb{Z}^{m}: k \cdot \alpha<d\right\}$. We have just mentioned that this has already been proved in [TB] when $H^{+}$and $H^{-}$are replaced by $H_{0}^{+}=\left\{k \in \mathbb{Z}^{m}: k \cdot \alpha \geq 0\right\}$ and $H_{0}^{-}=\left\{k \in \mathbb{Z}^{m}: k \cdot \alpha<0\right\}$. The idea of this proof is to translate the general case of $H^{+}$into the special case of $H_{0}^{+}$.

We will only prove that for $f \in L^{p}\left(\mathbb{T}^{m}\right), p \geq 2$, there holds the inequality

$$
\left\|f^{+}\right\|_{p} \leq p\|f\|_{p}
$$

where $f^{+}(x)=\sum_{k \in H^{+}} \hat{f}(k) e(k \cdot x)$. This result was already proved in TB] when $H^{+}$is replaced by $H_{0}^{+}$. The standard interpolation argument appearing in [TB] will then produce the first part of our conclusion; also the case of $H^{-}$can be treated in a very similar way.

Without loss of generality we may assume $|\alpha|=1$. Reversing $\alpha$ if necessary, we may also assume that $d>0$. First assume that $f$ has only a finite spectrum, i.e., that $f$ is a trigonometric polynomial. Move the halfspace $H_{0}^{+}$in the direction of $\alpha$ a distance of $d$ to make it coincide with $\mathrm{H}^{+}$. Then move it a little further if no point of the spectrum of $f$ lies on the boundary hyperplane until its edge first touches a point which is in the spectrum of $f$. Let $\ell$ be any such point. Observe that this maneuver might not have been possible if the spectrum of $f$ had been infinite, since there could have been no spectrum points on the boundary hyperplane but an infinite sequence of spectrum points approaching that hyperplane. Let $g(x)=$ $e(-\ell \cdot x) f(x)$. Since $|g|=|f|, g \in L^{p}$, by the result in [TB], $\sum_{m \cdot \alpha \geq 0} \hat{g}(m) e(m \cdot x)$ has controlled $L^{p}$ norm, $\left\|\sum_{m \cdot \alpha \geq 0} \hat{g}(m) e(m \cdot x)\right\|_{p} \leq p\|g\|_{p}$. But letting $m=$ $k-\ell$, this last sum becomes $e(-\ell \cdot x) \sum_{k \cdot \alpha \geq \ell \cdot \alpha} \hat{g}(k-\ell) e(k \cdot x)=e(-\ell \cdot x) f^{+}(x)$. Finally, $|e(-\ell \cdot x)|=1$, and $\left\|f^{+}(x)\right\|_{p} \leq p\|f\|_{p}$ follows.

But the inequality constant is independent of the spectrum size and the trigonometric polynomials are dense in $L^{p}$, so the general case of infinite spectrum follows by approximating $f$ by a sequence of trigonometric polynomials. We give the details. Define the square $(C, 1)$ to be this trigonometric polynomial

$$
\sigma_{n}(f)=\frac{1}{(n+1)^{m}} \sum_{i_{1}=0}^{n} \cdots \sum_{i_{m}=0}^{n} s_{i_{1} \ldots i_{m}}(f)
$$


where

$$
s_{i_{1} \ldots i_{m}}(f)=\sum_{k_{1}=-i_{1}}^{i_{1}} \ldots \sum_{k_{m}=-i_{m}}^{i_{m}} \hat{f}(k) e(k \cdot x) .
$$

Since $p \geq 2$, in particular $f \in L^{2}$, then from $\sum_{k \cdot \alpha \geq c}|\hat{f}(k)|^{2} \leq \sum|\hat{f}(k)|^{2}$ it follows that $f^{+} \in L^{2}$. It is well known [Z, Vol. II, Theorem 3.18] that since $f^{+} \in L^{2}$,

$$
\sigma_{n}\left(f^{+}\right) \rightarrow f^{+} \text {a.e. as } n \rightarrow \infty \text {. }
$$

Applying the finite spectrum inequality (2.8) to $\sigma_{n}(f)$, we get

$$
\begin{aligned}
\left\|\sigma_{n}\left(f^{+}\right)\right\|_{p} & =\left\|\left(\sigma_{n}(f)\right)^{+}\right\|_{p} \\
& \leq p\left\|\sigma_{n}(f)\right\|_{p} \\
& \leq p\|f\|_{p},
\end{aligned}
$$

where the last step was explained in the proof of part (3) of Lemma 2 above. Now let $n \rightarrow \infty$, using (2.9), the last inequality, and Fatou's lemma to get inequality (2.8) without any constraint on the spectrum of $f$. The case of $H^{-}$is exactly the same.

We may now pass to the proof of inequality (1.7).

Proof. Let $f \in L\left(\mathbb{T}^{m}\right)$ and let $W$ be a convex polyhedron. From equation (1.5), to prove inequality (1.7) it suffices to find a constant $C(m, p, W)$ so that $\left\|s_{N W}(f)\right\|_{p} \leq$ $C N^{m(1-1 / p)}\|f\|_{1}$. Pick an integer $D$ so large that condition (2.2) holds for $B=W$. First apply (2.4), then Lemma 4, then Lemma 3, and finally (2.5):

$$
\begin{aligned}
\left\|s_{N W}(f)\right\|_{p} & =\left\|s_{N W}\left(\tau_{2 D N}(f)\right)\right\|_{p} \\
& \leq \gamma\left(1+\frac{4 p^{2}}{p-1}\right)^{m}\left\|\tau_{2 D N}(f)\right\|_{p} \\
& \leq C \gamma\left(1+\frac{4 p^{2}}{p-1}\right)^{m} N^{m(1-1 / p)}\left\|\tau_{2 D N}(f)\right\|_{1} \\
& \leq 3^{m} C \gamma\left(1+\frac{4 p^{2}}{p-1}\right)^{m} N^{m(1-1 / p)}\|f\|_{1} \\
& =\gamma_{2}(W, m, p) N^{m(1-1 / p)}\|f\|_{1} .
\end{aligned}
$$

The following simple lemma allows us to convert an upper bound estimate for an $L^{p^{\prime}}$ norm of a Dirichlet kernel into a lower bound estimate of the $L^{p}$ norm of that kernel.

Lemma 5. If $S$ is any finite subset of $\mathbb{Z}^{m}$ of cardinality $n,|S|=n$, and if

$$
D=D(S)=\sum_{k \in S} e(k \cdot x),
$$

then

$$
\|D\|_{p} \geq \frac{n}{\|D\|_{p^{\prime}}}
$$

where $1 \leq p \leq \infty$ and $p$ and $p^{\prime}$ are conjugate exponents, $\frac{1}{p}+\frac{1}{p^{\prime}}=1$. 
Proof. Apply Plancherel's equation to get

$$
n=\sum_{k \in S} 1^{2}=\int_{\mathbb{T}^{m}}\left|\sum_{k \in S} e(k \cdot x)\right|^{2} d x=\int_{\mathbb{T}^{m}}|D D| d x
$$

and then Hölder's inequality:

$$
\int_{\mathbb{T}^{m}}|D D| d x \leq\left(\int_{\mathbb{T}^{m}}|D|^{p} d x\right)^{1 / p}\left(\int_{\mathbb{T}^{m}}|D|^{p^{\prime}} d x\right)^{1 / p^{\prime}}=\|D\|_{p}\|D\|_{p^{\prime}} .
$$

We can now give a quick proof of the lower bound inequality (1.8).

Proof. For every $p^{\prime}$ in $(1, \infty)$ we have

$$
\left\|D_{N W}\right\|_{p^{\prime}} \leq \gamma_{2}(W, m, p) N^{m\left(1-1 / p^{\prime}\right)} .
$$

From inequality (2.1), Lemma 5 , and this, we get

$$
\begin{aligned}
\left\|D\left(N W \cap \mathbb{Z}^{m}\right)\right\|_{p} & \geq \frac{d N^{m}}{\left\|D\left(N W \cap \mathbb{Z}^{m}\right)\right\|_{p^{\prime}}} \\
& \geq \frac{d N^{m}}{\gamma_{2}(W, m, p) N^{m\left(1-1 / p^{\prime}\right)}} \\
& =\frac{d}{\gamma_{2}(W, m, p)} N^{m\left(\frac{1}{p^{\prime}}\right)} \\
& =\gamma_{1}(W, m, p) N^{m(1-1 / p)},
\end{aligned}
$$

where $\gamma_{1}$ is defined to be $d / \gamma_{2}$. This establishes the lower bound inequality (1.8).

This completes our work on polyhedra. We move on to the proof of Theorem 3. The only geometric properties of bounded sets with non-empty interiors that we will need will be inequalities (2.1) and (2.2).

Proof. Since

$$
\frac{1}{p}=\frac{1}{2}+\frac{1}{\frac{2 p}{p+2}}-1
$$

Young's inequality gives

$$
\left\|D\left(N B \cap \mathbb{Z}^{m}\right) * T_{2 D N}(f)\right\|_{p} \leq\left\|D\left(N B \cap \mathbb{Z}^{m}\right)\right\|_{2}\left\|T_{2 D N} f\right\|_{\frac{2 p}{p+2}},
$$

where the requirement that $\frac{2 p}{p+2} \geq 1$ follows from $p \geq 2$. Since $T_{2 D N}(f)$ has at most $O\left(N^{m}\right)$ terms, we have the change of norm inequality

$$
\begin{aligned}
\left\|T_{2 D N}(f)\right\|_{\frac{2 p}{p+2}} & \leq C\left\|T_{2 D N}(f)\right\|_{1}\left(N^{m}\right)^{\frac{1}{1}-\frac{1}{\frac{2 p}{p+2}}} \\
& =C\left(N^{m}\right)^{\frac{1}{2}-\frac{1}{p}}\left\|T_{2 D N}(f)\right\|_{1} .
\end{aligned}
$$

We also use property (2.5) of the delayed means

$$
\left\|T_{2 D N}(f)\right\|_{1} \leq 3^{m}\|f\|_{1} .
$$

The final item is Plancherel's Theorem,

$$
\left\|D\left(N B \cap \mathbb{Z}^{m}\right)\right\|_{2}=\left(\sum_{k \in N B \cap \mathbb{Z}^{m}} 1\right)^{1 / 2} \leq C N^{\frac{m}{2}} .
$$


Putting together inequalities (2.11) to (2.14) gives

$$
\begin{aligned}
\left\|D\left(N B \cap \mathbb{Z}^{m}\right) * f\right\|_{p} & \leq\left\|D\left(N B \cap \mathbb{Z}^{m}\right)\right\|_{2}\left\|T_{2 D N}(f)\right\|_{\frac{2 p}{p+2}} \\
& \leq\left\|D\left(N B \cap \mathbb{Z}^{m}\right)\right\|_{2} C\left(N^{m}\right)^{\frac{1}{2}-\frac{1}{p}}\left\|T_{2 D N}(f)\right\|_{1} \\
& \leq C N^{\frac{m}{2}} 3^{m} N^{m\left(\frac{1}{2}-\frac{1}{p}\right)}\|f\|_{1} \\
& =\gamma_{2} N^{m\left(1-\frac{1}{p}\right)}\|f\|_{1} .
\end{aligned}
$$

Theorem 4 is immediate from this and Lemma 5

Proof. We are assuming that $p \in(1,2]$ so that $p^{\prime} \in[2, \infty)$ and that $B$ is bounded with non-empty interior. We simply repeat the proof of inequality (1.8) given above, except that $p^{\prime}$ is constrained to $[2, \infty)$ and $W$ is replaced by $B$.

We next prove Theorem 6 .

Proof. We are assuming that $B$ is bounded, has non-empty interior and satisfies the $M_{N}$-condition. We need to show that for every $p \geq 2$, the $L^{p}\left(\mathbb{T}^{m}\right)$ norm of $D_{N B}(x)$ is bounded below by $\gamma_{1} N^{m(1-1 / p)}$. We take inspiration from the paper of Liflyand $\mathrm{L}$. Let $A_{N B}$ be a union of cubes of side 1 whose center is at points of $N B \cap \mathbf{Z}^{m}$. Let $Q(z)$ be the cube of side 1 centered at $z$. The Fourier transform of the characteristic function of $Q(0)$, the cube of side 1 centered at the origin, is $\int_{Q(0)} e(-x \cdot z) d z=\int_{Q(0)} e^{-2 \pi i x \cdot z} d z=\prod_{j=1}^{m} \frac{\sin \left(\pi x_{j}\right)}{\pi x_{j}}$, and so the Fourier transform of the characteristic function of $Q(z)=z+Q(0)$ is $e(-z \cdot x) \prod_{j=1}^{m} \frac{\sin \left(\pi x_{j}\right)}{\pi x_{j}}$. Then,

$$
\begin{aligned}
& \int_{A_{N B}} e(-u \cdot x) d u=\sum_{z \in N B \cap \mathbf{Z}^{m}} \int_{Q(z)} e(-u \cdot x) d u \\
& =\prod_{j=1}^{m} \frac{\sin \left(\pi x_{j}\right)}{\pi x_{j}} \sum_{z \in N B \cap \mathbf{Z}^{m}} e(-z \cdot x)=\prod_{j=1}^{m} \frac{\sin \left(\pi x_{j}\right)}{\pi x_{j}} \overline{D_{N B}(x)},
\end{aligned}
$$

and we have proved the following important identity:

$$
\int_{A_{N B}} e(-u \cdot x) d u=\prod_{j=1}^{m} \frac{\sin \left(\pi x_{j}\right)}{\pi x_{j}} \overline{D_{N B}(x)} .
$$

By the elementary inequality $\frac{2}{\pi} \leq \frac{\sin x}{x} \leq 1$ which is valid when $|x| \leq \frac{\pi}{2}$, we can see from $|\bar{a}|=|a|$ that

$$
\left\|D_{N B}\right\|_{p} \geq\left(\int_{\mathbb{T}^{m}}\left|\int_{A_{N B}} e(-u \cdot x) d u\right|^{p} d x\right)^{\frac{1}{p}}=\left\|\hat{\chi}_{A_{N B}}\right\|_{p} .
$$

Integrating $e(-u \cdot x)$ over $A_{N B}$ and noting that $A_{N B}=N B \backslash\left(N B \backslash A_{N B}\right) \cup$ $\left(A_{N B} \backslash N B\right)$ gives

$$
\hat{\chi}_{A_{N B}}(x)=\hat{\chi}_{N B}(x)-\hat{\chi}_{N B \backslash A_{N B}}(x)+\hat{\chi}_{A_{N B} \backslash N B}(x) .
$$


Take the $L^{p}\left(\mathbb{T}^{m}\right)$ norm of $e(-u \cdot x)$ and apply the triangle inequality to get

$$
\left\|\hat{\chi}_{A_{N B}}\right\|_{p} \geq\left\|\hat{\chi}_{N B}\right\|_{p}-\left\|\hat{\chi}_{N B \backslash A_{N B}}\right\|_{p}-\left\|\hat{\chi}_{A_{N B} \backslash N B}\right\|_{p} .
$$

Note that we reserve $\|\cdot\|_{p}$ for $\|\cdot\|_{L^{p}\left(\mathbb{T}^{m}\right)}$; however, we will always write $\|\cdot\|_{L^{p}\left(\mathbb{R}^{m}\right)}$ without abbreviation. Since $T^{m} \subset R^{m}$, we have

$$
\left\|\hat{\chi}_{N B \backslash A_{N B}}\right\|_{p}=\left\|\hat{\chi}_{N B \backslash A_{N B}}\right\|_{L^{p}\left(\mathbb{T}^{m}\right)} \leq\left\|\hat{\chi}_{N B \backslash A_{N B}}\right\|_{L^{p}\left(\mathbb{R}^{m}\right)},
$$

and since $p \geq 2$, from the Hausdorff-Young inequality there follows

$$
\left\|\hat{\chi}_{N B \backslash A_{N B}}\right\|_{L^{p}\left(\mathbb{R}^{m}\right)} \leq\left\|\chi_{N B \backslash A_{N B}}\right\|_{L^{p^{\prime}\left(\mathbb{R}^{m}\right)}}=\left|N B \backslash A_{N B}\right|^{1-\frac{1}{p}},
$$

so that

$$
\left\|\hat{\chi}_{N B \backslash A_{N B}}\right\|_{p} \leq\left|N B \backslash A_{N B}\right|^{1-\frac{1}{p}} .
$$

The same reasoning also produces

$$
\left\|\hat{\chi}_{A_{N B} \backslash N B}\right\|_{p} \leq\left|A_{N B} \backslash N B\right|^{1-\frac{1}{p}} .
$$

A change of variables yields

$$
\begin{aligned}
& \left\|\hat{\chi}_{N B}\right\|_{p}=\left(\int_{\mathbb{T}^{m}}\left|\hat{\chi}_{N B}(x)\right|^{p} d x\right)^{\frac{1}{p}}=N^{m(1-1 / p)}\left(\int_{N \mathbb{T}^{m}}\left|\hat{\chi}_{B}(x)\right|^{p} d x\right)^{\frac{1}{p}} \\
& =N^{m(1-1 / p)}\left(\left\|\hat{\chi}_{B}\right\|_{L^{p}\left(\mathbb{R}^{m}\right)}-\left\|\hat{\chi}_{B}\right\|_{L^{p}\left(\mathbb{R}^{m} \backslash N \mathbb{T}^{m}\right)}\right) .
\end{aligned}
$$

Combine this with relations (2.16), (2.17), (2.18), and (2.19) to get

$$
\left\|D_{N B}\right\|_{p} \geq N^{m(1-1 / p)}\left(\begin{array}{c}
\left\|\hat{\chi}_{B}\right\|_{L^{p}\left(\mathbb{R}^{m}\right)}-\left\|\hat{\chi}_{B}\right\|_{L^{p}\left(\mathbb{R}^{m} \backslash N \mathbb{T}^{m}\right)} \\
-\left\{\frac{\left|N B \backslash A_{N B}\right|^{1-\frac{1}{p}}+\left|A_{N B} \backslash N B\right|^{1-\frac{1}{p}}}{N^{m(1-1 / p)}}\right\}
\end{array}\right) .
$$

The Hausdorff-Young inequality implies that $\hat{\chi}_{B} \in L^{p}\left(\mathbb{R}^{m}\right)$. (In fact, $\left\|\hat{\chi}_{B}\right\|_{L^{p}\left(\mathbb{R}^{m}\right)}$ $\leq|B|^{1-\frac{1}{p}}$.) So by Lebesgue's dominated convergence theorem, $\left\|\hat{\chi}_{B}\right\|_{L^{p}\left(\mathbb{R}^{m} \backslash N \mathbb{T}^{m}\right)}$ tends to 0 as $N \rightarrow \infty$. Because of the $M_{N}$ condition, both $\left|N B \backslash A_{N B}\right|$ and $\left|A_{N B} \backslash N B\right|$ are $o\left(N^{m}\right)$, so the term in curly brackets also goes to zero when $N \rightarrow \infty$. Thus from inequality (2.20) it follows that there is a constant $\gamma_{1}$ so that

$$
\left(\int_{\mathbb{T}^{m}}\left|D_{N B}(x)\right|^{p} d x\right)^{\frac{1}{p}} \geq \gamma_{1} N^{m(1-1 / p)}
$$

Remark 2. This proof showed that $\left\|\hat{\chi}_{N B}\right\|_{p}$ is asymptotic to $c N^{m(1-1 / p)}$ where $c=\left\|\hat{\chi}_{B}\right\|_{L^{p}\left(\mathbb{R}^{m}\right)}$. Since inequality (2.16) can be reversed (with constant $(\pi / 2)^{m}$ instead of 1 ), an upper bound for the case of $L^{p}$ requires no extra work. However, this is not useful, since the case for an upper bound when $p \geq 2$ was already done without the boundary assumption $M_{N}$ in the proof of Theorem 3 


\section{Proof of Theorem 5}

We will only estimate the value of $\left\|D_{N B}\right\|_{p}$ where $B=\left\{x \in R^{m}:|x| \leq 1\right\}$ from below. More specifically, we will show that

$$
\left\|D_{N B}\right\|_{p} \geq\left\{\begin{array}{cc}
\gamma_{1} N^{\frac{m-1}{2}}, & 1 \leq p<\frac{2 m}{m+1} \\
\gamma_{1} N^{\frac{m-1}{2}} \ln ^{1 / p} N, & p=\frac{2 m}{m+1}
\end{array}\right.
$$

We will prove this lemma below.

Lemma 6. For every $p \in\left[1, \frac{2 m}{m+1}\right)$ there is a positive constant $c_{1}(p, m)$ so that for all $R>1$,

$$
\int_{R B}\left|\int_{B} e(-v \cdot y) d v\right|^{p} d y \geq c_{1}^{p} R^{-\frac{m+1}{2} p+m}
$$

If $p=\frac{2 m}{m+1}$, there is a positive constant $c_{1}(m)$ so that for all $R>1$,

$$
\int_{R B}\left|\int_{B} e(-v \cdot y) d v\right|^{p} d y \geq c_{1}^{p} \ln R
$$

Let $p \in\left[1, \frac{2 m}{m+1}\right)$ and assume temporarily the validity of inequality (3.1).

For a small positive $\epsilon<1 / 2$ (to be chosen later) we have

$$
\left\|D_{N B}\right\|_{p}=\left(\int_{\mathbb{T}^{m}}\left|D_{N B}\right|^{p} d x\right)^{1 / p} \geq\left(\int_{\epsilon B}\left|D_{N B}\right|^{p} d x\right)^{1 / p} .
$$

Here, the same reasoning that led from identity (2.15) to inequalities (2.16) and (2.17) above produces

$$
\begin{aligned}
\left(\int_{\epsilon B}\left|D_{N B}\right|^{p} d x\right)^{1 / p} & \geq\left(\int_{\epsilon B}\left|\widehat{\chi_{N B}}(x)\right|^{p} d x\right)^{\frac{1}{p}}-\left(\int_{\epsilon B}\left|\chi_{A_{N B}-N B}(x)\right|^{p} d x\right)^{\frac{1}{p}} \\
& -\left(\int_{\epsilon B}\left|\chi_{N B-A_{N B}}(x)\right|^{p} d x\right)^{\frac{1}{p}} .
\end{aligned}
$$

Concatenating the last two inequalities,

$$
\begin{aligned}
\left\|D_{N B}\right\|_{p} & \geq\left(\int_{\epsilon B}\left|\widehat{\chi_{N B}}(x)\right|^{p} d x\right)^{\frac{1}{p}} \\
& -\left\{\left(\int_{\epsilon B}\left|\chi_{A_{N B}-N B}(x)\right|^{p} d x\right)^{\frac{1}{p}}+\left(\int_{\epsilon B}\left|\chi_{N B-A_{N B}}(x)\right|^{p} d x\right)^{\frac{1}{p}}\right\} .
\end{aligned}
$$


First making the change of variable $u=N v, d u=N^{m} d v$ and then the substitution $y=N x, d y=N^{m} d x$, we estimate the first term on the right hand side of (3.3):

$$
\begin{aligned}
\left(\int_{\epsilon B}\left|\widehat{\chi_{N B}}(x)\right|^{p} d x\right)^{\frac{1}{p}} & =\left(\int_{\epsilon B}\left|\int_{N B} e(-u \cdot x) d u\right|^{p} d x\right)^{\frac{1}{p}} \\
& =\left(\int_{\epsilon B} N^{m p}\left|\int_{B} e(-v \cdot N x) d v\right|^{p} d x\right)^{\frac{1}{p}} \\
& =N^{m\left(1-\frac{1}{p}\right)}\left(\int_{\epsilon N B}\left|\int_{B} e(-v \cdot y) d v\right|^{p} d y\right)^{\frac{1}{p}} \\
& \geq N^{m\left(1-\frac{1}{p}\right)}\left(c_{1}^{p}(\epsilon N)^{-\frac{m+1}{2} p+m}\right)^{\frac{1}{p}} \\
& =c_{1} N^{\frac{m-1}{2}} \epsilon^{m\left(\frac{1}{p}-\frac{1}{2}\right)-\frac{1}{2}} .
\end{aligned}
$$

The last inequality follows from the estimate (3.1).

Since $p<2$ we may estimate each of the last two terms in (3.3) by applying Hölder's inequality with exponents $2 / p$ and $2 /(2-p)$, then expanding the domain of integration to $R^{n}$, and then applying the Plancherel equation. We get

$$
\begin{aligned}
\left(\int_{\epsilon B}\left|\chi \widehat{A_{N B}-N B}(x)\right|^{p} d x\right)^{\frac{1}{p}} & \leq\left(\int_{\epsilon B}\left|\chi \widehat{A_{N B}-N B}(x)\right|^{2} d x\right)^{\frac{1}{2}}\left(v_{m} \epsilon^{m}\right)^{\frac{2-p}{2 p}} \\
& =\left|A_{N B}-N B\right|^{\frac{1}{2}} v_{m}^{\left(\frac{1}{p}-\frac{1}{2}\right)} \epsilon^{m\left(\frac{1}{p}-\frac{1}{2}\right)}
\end{aligned}
$$

and

$$
\left(\int_{\epsilon B}\left|\chi_{N B-A_{N B}}(x)\right|^{p} d x\right)^{\frac{1}{p}} \leq\left|N B-A_{N B}\right|^{\frac{1}{2}} v_{m}^{\left(\frac{1}{p}-\frac{1}{2}\right)} \epsilon^{m\left(\frac{1}{p}-\frac{1}{2}\right)}
$$

where $v_{m}=\omega_{m} / m=2 \pi^{m / 2} /(m \Gamma(m / 2))$ is the volume of the $m$-dimensional ball. Because of the geometry of the $m$-sphere, we know that

$$
\left|A_{N B}-N B\right|^{\frac{1}{2}}+\left|N B-A_{N B}\right|^{\frac{1}{2}}=O\left(N^{\frac{m-1}{2}}\right)([\underline{\mathrm{S}}]) \text {. }
$$

Thus there is a constant $c_{2}(p, m)$ so that the quantity in curly brackets in inequality (3.3) is $\leq c_{2} \epsilon^{m\left(\frac{1}{p}-\frac{1}{2}\right)} N^{\frac{m-1}{2}}$. From (3.3), this, and inequality (3.4) we get

$$
\begin{aligned}
\left\|D_{N B}\right\|_{p} & \geq c_{1} N^{\frac{m-1}{2}} \epsilon^{m\left(\frac{1}{p}-\frac{1}{2}\right)-\frac{1}{2}}-c_{2} \epsilon^{m\left(\frac{1}{p}-\frac{1}{2}\right)} N^{\frac{m-1}{2}} \\
& =\epsilon^{m\left(\frac{1}{p}-\frac{1}{2}\right)-\frac{1}{2}}\left(c_{1}-\epsilon^{\frac{1}{2}} c_{2}\right) N^{\frac{m-1}{2}} \\
& =\gamma_{1} N^{\frac{m-1}{2}} .
\end{aligned}
$$

We now choose $\epsilon$. Choose it so small that $\gamma_{1}>0$.

If $p=\frac{2 m}{m+1}$, we do not need the $\epsilon$ scaling trick. Let $\epsilon=1$. Then inequality (3.3) becomes

$$
\begin{aligned}
\left\|D_{N B}\right\|_{p} & \geq\left(\int_{B}\left|\widehat{\chi_{N B}}(x)\right|^{p} d x\right)^{\frac{1}{p}} \\
& -\left\{\left(\int_{B}\left|\chi_{A_{N B}-N B}(x)\right|^{p} d x\right)^{\frac{1}{p}}+\left(\int_{B}\left|\chi_{N_{B-A_{N B}}}(x)\right|^{p} d x\right)^{\frac{1}{p}}\right\},
\end{aligned}
$$


so that

$$
\left\|D_{N B}\right\|_{p} \geq\left(\int_{B}\left|\widehat{\chi_{N B}}(x)\right|^{p} d x\right)^{\frac{1}{p}}-c_{2}^{\prime} N^{\frac{m-1}{2}}
$$

and using estimate (3.2) instead of estimate (3.1) allows us to replace inequality (3.4) with

$$
\begin{aligned}
\left(\int_{B}\left|\widehat{\chi_{N B}}(x)\right|^{p} d x\right)^{\frac{1}{p}} & \geq\left(\int_{\epsilon B}\left|\int_{N B} e(-u \cdot x) d u\right|^{p} d x\right)^{\frac{1}{p}} \\
& =c_{1} N^{\frac{m-1}{2}} \ln N .
\end{aligned}
$$

Putting this together with (3.6a) gives (3.2). It only remains to prove the lemma.

Proof. It is well known that

$$
\widehat{\chi_{B}}(x)=\int_{|\xi| \leq 1} e(-x \cdot \xi) d x=J_{m / 2}(2 \pi|x|)|x|^{-m / 2},
$$

where $J_{\nu}$ is the standard Bessel function of order $\nu$. (See Stein \& Weiss, p. 170, Thm. 4 with $\delta=0$.) So we have to estimate

$$
\begin{aligned}
\int_{R B}\left|\widehat{\chi_{B}}(x)\right|^{p} d x & =\int_{R B}\left|\int_{B} e(-v \cdot y) d v\right|^{p} d y \\
& =\omega_{m} \int_{0}^{R}\left|J_{m / 2}(2 \pi r)\right|^{p} r^{-m p / 2} r^{m-1} d r .
\end{aligned}
$$

Since $J_{m / 2}(r)$ is asymptotic to $O\left(r^{m / 2}\right)$ for small $r$, the integrand is bounded for small $r$, so a lower estimate of the form $c R^{\beta}$ with $\beta>0$ will hold equally well for this integral or for

$$
I=(2 \pi)^{m-m p / 2} \int_{a}^{R}\left|J_{m / 2}(2 \pi r)\right|^{p} r^{-m p / 2+m-1} d r,
$$

where $a$ is any fixed positive constant. In other words, to prove the lemma it suffices to find a positive $c_{1}(p, m)$ for which there holds

$$
I \geq \begin{cases}c_{1}^{p} R^{-\frac{m+1}{2} p+m} & \text { if } 1 \leq p<\frac{2 m}{m+1}, \\ c_{1}^{p} \ln R & \text { if } p=\frac{2 m}{m+1} .\end{cases}
$$

In the integral defining $I$, substitute $s=2 \pi r$ to write

$$
I=\int_{2 \pi a}^{2 \pi R}\left|J_{m / 2}(s)\right|^{p} s^{m-m p / 2-1} d s .
$$

A well known large variable estimate for Bessel functions is that

$$
J_{m / 2}(s)=\sqrt{\frac{2}{\pi}} \frac{\cos \left(s-\frac{m+1}{4} \pi\right)}{s^{1 / 2}}+O\left(\frac{1}{s^{3 / 2}}\right)([\mathrm{E}]) .
$$

It follows from this that

$$
I=\sqrt{\frac{2}{\pi}} J+E
$$

where

$$
J=\int_{2 \pi a}^{2 \pi R}\left|\frac{\cos \left(s-\frac{m+1}{4} \pi\right)}{s^{1 / 2}}\right|^{p} s^{m-m p / 2-1} d s
$$


and $E$ is on the order of

$$
\begin{aligned}
\int_{2 \pi a}^{2 \pi R}\left|\frac{1}{s^{3 / 2}}\right|^{p} s^{m-m p / 2-1} d s & =O\left(R^{m-\frac{m+1}{2} p-p}\right) \\
& =o\left(R^{m-\frac{m+1}{2} p}\right) .
\end{aligned}
$$

We will underestimate $J$ by replacing $\cos \left(s-\frac{m+1}{4} \pi\right)$ by $\frac{1}{2}$ when it is greater than or equal to $\frac{1}{2}$ and by 0 elsewhere. Specifically, if

$$
k \in\left[\left[a+\frac{m+1}{8}+\frac{1}{6}\right\rceil,\left\lfloor R+\frac{m+1}{8}-\frac{1}{6}\right\rfloor\right]=[b, t],
$$

where $\lceil\alpha\rceil$ denotes the least integer $\leq \alpha$ and $\lfloor\alpha\rfloor$ denotes the greatest integer $\geq \alpha$, then

$$
I_{k}=\left[2 \pi k-\frac{m+1}{4} \pi-\frac{\pi}{3}, 2 \pi k-\frac{m+1}{4} \pi+\frac{\pi}{3}\right] \subset[2 \pi a, 2 \pi R]
$$

and for $s \in I_{k}, \cos \left(s-\frac{m+1}{4} \pi\right) \geq \frac{1}{2}$. Hence

$$
\begin{aligned}
J & \geq \sum_{k=b}^{t} \int_{I_{k}} \frac{\left(\frac{1}{2}\right)^{p}}{s^{p / 2}} s^{m-m p / 2-1} d s \\
& =\left(\frac{1}{2}\right)^{p} \sum_{k=b}^{t} \int_{I_{k}} s^{\alpha-1} d s,
\end{aligned}
$$

where $\alpha=\frac{m+1}{2}\left(\frac{2 m}{m+1}-p\right)$ so that $\alpha-1>-1$. Underestimate each integrand by its smallest value to get

$$
J \geq\left(\frac{1}{2}\right)^{p} \sum_{k=b}^{t}\left(2 \pi k-\frac{m+1}{4} \pi+\operatorname{sgn}(\alpha-1) \frac{\pi}{3}\right)^{\alpha-1}\left(2 \frac{\pi}{3}\right) .
$$

Finally note that $b$ is bounded, $t=R-c$ where $c$ is bounded, and use the integral test estimate of comparing $\sum f(k)$ with $\int f(k)$ for positive monotone $f$ to see that there is a positive constant $d_{1}(p, m)$ so that

$$
J \geq \begin{cases}d_{1} R^{\alpha}=R^{-\frac{m+1}{2} p+m} & \text { if } 1 \leq p<\frac{2 m}{m+1}, \\ d_{1} \ln R & \text { if } p=\frac{2 m}{m+1} .\end{cases}
$$

Putting this estimate and estimate (3.10) into equation (3.9) allows us to complete estimate (3.7) and thereby finish the proof.

\section{REFERENCES}

[A] J. M. Ash, Triangular Dirichlet kernels and growth of $L^{p}$ Lebesgue constants, preprint. [AAJRS] B. Anderson, J. M. Ash, R. Jones, D. G. Rider, B. Saffari, Exponential sums with coefficients 0 or 1 and concentrated $L^{p}$ norms, to appear in Annales de l'Institut Fourier.

[C] C. K. Chui, An introduction to wavelets. Wavelet Analysis and its Applications, 1. Academic Press, Inc., Boston, MA, 1992. MR1150048 (93f:42055)

[E] A. Erdélyi et al., Higher transcendental functions. Vol. II. Based, in part, on notes left by Harry Bateman. McGraw-Hill, New York-Toronto-London, 1953. Page 85, 7.13.1(3).

[GKP] R. L. Graham, D. E. Knuth, O. Patashnik, Concrete mathematics. A foundation for computer science. Second edition. Addison-Wesley Publishing Company, Reading, MA, 1994. MR:1397498 (97d:68003) 
[I] V. A. Ilyin, Problems of localization and convergence for Fourier series in fundamental systems of the Laplace operator, Uspekhi Mat. Nauk 23(1968), 61-120. (Translation in Russian Mathematical Surveys 23, 59-116.) MR0223823 (36:6870)

[K] A. Ya. Khinchin, Continued Fractions, Nauka, Moscow (1978). Also translated from the third (1961) Russian edition. Reprint of the 1964 translation. Dover Publications, Inc., Mineola, NY, 1997. MR.1451873 (98c:11008)

[L] E. R. Liflyand, Lebesgue constants of multiple Fourier series, Online Journal of Analytic Combinatorics, Issue 1 (2006), \# 5, 1-112. MR2249993 (2007g:42017)

[NP] F. L. Nazarov and A. N. Podkorytov, On the behavior of the Lebesgue constants of twodimensional Fourier sums over polygons, St. Petersburg Math. J. 7 (1996), 663-680. MR.1356537 (96m:42019)

[NZ] Niven and Zuckerman, An introduction to the theory of numbers, 3rd edition, Wiley, 1972. MR0344181(49:8921)

[Sh] H. S. Shapiro, Lebesgue constants for spherical partial sums, J. of Approximation theory 13(1975), 40-44. MR0374808 (51:11004)

[S] E M. Stein, Harmonic analysis: real-variable methods, orthogonality, and oscillatory integrals. With the assistance of Timothy S. Murphy. Princeton Mathematical Series, 43. Monographs in Harmonic Analysis, III. Princeton University Press, Princeton, NJ, 1993, pp. 362-363. MR 1232192 (95c:42002)

[SW] E. M. Stein and G. Weiss, Introduction to Fourier analysis on Euclidean spaces, Princeton Mathematical Series, No. 32. Princeton Univ. Press, Princeton, N.J., 1971. MR.0304972 (46:4102)

[TB] R. M. Trigub and E. S. Belinsky, Fourier analysis and approximation of functions, Kluwer Academic Publishers, Dordrecht, 2004. MR2098384 (2005k:42002)

[Y] V. A. Yudin, Behavior of Lebesgue constants, Translated from Matematicheskie Zametki, 17(1975), 401-405. (Translation is in Mathematical Notes 17 on pages 233-235.) MR0417690(54:5739)

[YY] A. A. Yudin and V. A. Yudin, Polygonal Dirichlet kernels and growth of Lebesgue constants, Translated from Matematicheskie Zametki, 37(1985), 220-236. (Translation is in Mathematical Notes 37 on pages 124-135.) MR784367(86k:42039)

[Z] A. Zygmund, Trigonometric Series, v.1, 2nd ed., Cambridge University Press, New York, 1959. MR0107776(21:6498)

Department of Mathematics, DePaul University, Chichgo, Illinois 60614

E-mail address: mash@math.depaul.edu

$U R L:$ http://www.depaul.edu/ $\sim$ mash

Department of Mathematics, Florida International University, University Park, MiAMI, FLORIDA 33199

E-mail address: decarlil@fiu.edu

URL: http://www.fiu.edu/ $\sim$ decarlil 